\title{
Cardiotoxicity: An Unusual Case of Methotrexate Overdose
}

\author{
R Nhiri*, S Bellamqddam, S Amaqdouf and N Elouafi \\ Department of Cardiology, University Hospital of Mohammed VI, University Mohammed the First, Oujda, Morocco.
}

*Corresponding author: R Nhiri, Department of Cardiology, University Hospital of Mohammed VI, University Mohammed the First, Oujda, Morocco.

Received Date: September 23, 2020

Published Date: December 18, 2020

\begin{abstract}
The methotrexate (MTX) is an antimetabolite, whose dosages vary according to indication. It is used in the treatment of tumoral pathologies, as acute lymphoblastic leukaemias and in rheumatology, in the rheumatoid polyarthritis (RP) and other chronic inflammatory rheumatisms. The methotrexate belongs to the group of antifolates: it inhibits purine and pyrimidine synthesis, which accounts for its efficacy in the therapy of cancer as well as for some of its toxicities. Relative or absolute overdoses in low-dose methotrexate treatments for non-oncological diseases are regularly reported, either in isolated cases or in small series. The bone marrow toxicity with thrombocytopenia and leuconeutropenia is most often the first sign of general involvement. The cardiotoxicity of methotrexate is very rare, in this article we report the observation of pancytopenia associated with cardiotoxicity in a woman treated for RA, for whom the etiological investigation revealed inadvertent methotrexate intoxication by mistake of dosage.
\end{abstract}

\section{Introduction}

Methotrexate is a cytotoxic of the antifolate family; it is widely prescribed in oncohaematology but also in rheumatology, particularly in the treatment of certain autoimmune and inflammatory diseases, in particular rheumatoid arthritis. It acts as a false substrate, competitively inhibits the activity of dihydrofolate reductase (DHFR), a key enzyme in folate metabolism, and induces a deficiency of purine and pyrimidine bases, leading to a defect of DNA synthesis and Cell cycle blockade in S phase [1]. In tumoral pathologies, the administration of MTX is daily, and at high doses, whereas it is weekly in rheumatoid arthritis [2]. Thus, the toxicity of methotrexate is essentially obtained at anti-cancer doses, but relative or absolute overdoses in the context of low-dose methotrexate treatments for non-oncological diseases are regularly reported, either in isolated cases or in the form of small series. The toxicity of methotrexate is polymorphic, it is dose-dependent, Different organs can be affected [3]. We report the observation of cardiotoxicity in a woman treated for RA, in whom the etiological investigation revealed methotrexate involuntary intoxication by mistake of dosage.

\section{Observation}

A 68-year-old woman hypertensive, diabetic, followed for atrial fibrillation (AF) for 15 years, known carrier of a PR since 6 years, treated with methotrexate $5 \mathrm{mg} /$ week. The patient presented to the emergency department for rectorragies of low abundance, the biological assessment objectified pancytopenia for which she was hospitalized in the internal medicine department; the whole of the infectious workup including a cytobacteriological urine exam, a blood culture, viral serologies, and a chest X-ray, as well as the digestive explorations (FOGD, colonoscopy, abdominal angioscan) were without particularities; the myelogram revealed an absence of blocking of maturation of the blood lines, a megaloblastoid aspect of the erythroblasts and a gigantism of some granulocytes compatible 
with a toxic attack of the marrow. The drug investigation found a recent renewal of the prescription. However, the patient had changed the frequency of administration of methotrexate for seven days, from $5 \mathrm{mg}$ per week to $5 \mathrm{mg}$ per day with occasional selfmedication with NSAIDs. The hypothesis of iatrogenic pancytopenia was mentioned. Therapy with methotrexate was discontinued.

During her hospitalization, the patient presented a clinical symptoms of leftheartfailure, in which echocardiographic evaluation revealed severe LV dysfunction with LVEF at 25\%, while LV function was preserved on previous echocardiographic examinations. Symptomatic treatment, with the administration of folinic acid and platelet and blood transfusion support was undertaken, allowing almost complete improvement in clinical status and normalization of the biological status. The echocardiographic control realized two months later, objectified a LVEF at 51\% Given the reversible character of LV dysfunction after stopping methotrexate, and the absence of other etiologies explaining this dysfunction, the diagnosis of methotrexate cardiotoxicity was retained.

\section{Discussion}

Methotrexate (antineoplastic cytostatic) belongs to the group of antifolates: it is an analogue of vitamin B9 or folic acid, whose main mode of action and its transformation with additions of glutamyl radicals (MTXGlu) condition his activity. The digestive absorption is variable according to the dosage of MTX and from one patient to another. It must be remembered that 45 to $55 \%$ of MTXGlu is protein-bound, and in case of undernutrition the free form is predominant; the catabolism is hepatic [2]. This enzyme makes it possible to reduce dihydrofolic acid to different tetrahydrofolic acids. Elimination is mainly renal. Regardless of the route of administration, the passage of methotrexate into the blood and tissues is very rapid. The plasma half-life in adults is of the order of 2 hours, with protein binding of the order of $50 \%$ to $70 \%[4,5]$. The optimal dose (maximum $25 \mathrm{mg} /$ week) should be reached within 4 to 8 weeks [6]. Risk factors for methotrexate toxicity include: a relatively high dose of MTX, renal impairment, nonsteroidal antiinflammatory drugs, an age over 55, folate deficiency, low serum albumin, and drug interactions [7-9].

Non-steroidal anti-inflammatory drugs (NSAIDs) influence the pharmacokinetics of methotrexate. Some may lower renal excretion of methotrexate by inhibiting renal transport proteins and decreasing renal perfusion. Concomitant administration of NSAIDs may thus be associated with an increased risk of toxicity, as in this case, overdosage with methotrexate is combined with taking NSAIDs [10]. For our patient, the interrogation revealed the concept of self-medication with NSAIDs and a daily dose of $5 \mathrm{mg} /$ day of methotrexate for one week. The toxicity of MTX manifests itself in several forms, including hepatotoxicity, pulmonary toxicity, acute renal failure, stomatitis, ulceration/erosions of the gastrointestinal tract, and pancytopenia [11-13], cardiotoxicity is very rare.
We report the observation of methotrexate-induced cardiotoxicity induced by methotrexate overdose as a result of dosage error, this manifestation is unusual, and literature data for cardiotoxicity induced by methotrexate are very poor. The reported case is an unusual and rare form of methotrexate toxicity, the diagnosis of cardiac toxicity was retained ahead the installation of symptoms of acute heart failure and the discovery of a LV dysfunction at $25 \%$ in the context of a methotrexate overdose in a patient until there asymptomatic at the cardiac level, with left ventricular function preserved before hospitalization for methotrexate intoxication, and the reversibility of this dysfunction after discontinuation of methotrexate, objectified on echocardiographic control performed two month after treatment interruption. The implementation of MTX treatment must be accompanied by clear information to the patient, or even an education program.

Our investigation showed a recent renewal of the prescription by the treating doctor. However, the increase in the frequency of methotrexate intake, from weekly to daily, has led to this intoxication. When dispensing a medication prescribed by a doctor, the pharmacist is required to check the validity of the prescription, to analyze its contents and to check the treatment modalities (doses, routes of administration, duration), contraindications and interactions. If in doubt, he should contact the prescriber to verify the prescription and then explain to the patient the details of the treatment and, if necessary, the precautions to be taken and the possible adverse effects. This information must, of course, be given with discretion, respecting professional secrecy. We found that this filter certainly did not work well.

\section{Acknowledgement}

None.

\section{Conflict of interest}

The authors did not declare any conflict of interest regarding this article.

\section{References}

1. S Reutenauer, D Chauveau, C Récher (2009) Methotrexate overdose: complications, management and prevention. Intensive care 18: 654658.

2. Nadine SIMO, Callixte KUATE TEGUEU, David CHAURy, Mbole RANAIVO, Mam RAKOTO, et al. (2014) Methotrexate Iatrogenicity: A Shared Responsibility. Rev Geriatr 39(4): 265-269.

3. Mouna Lamchahab, Said Benchekroun (2014) Methotrexate toxicity in acute lymphoblastic leukemia. Pan Afr Med J 17: 16.

4. Eyriere H, Cociglio M, Margueritte G, Vallat C, Blayac JP, et al. (2004) Optimal management of methotrexate intoxication in a child with osteosarcoma. Ann Pharmacother 38(3): 422-427.

5. Maignen F, Guillot E, Pierron E, Julian S, Castot A (1996) Acute methotrexate poisoning: about 16 cases reports to the Paris AntiPoisons Center in review of the literature. Therapy 51: 527-531.

6. Aurore Le Quellec, Guillermo Carvajal Alegria, Dewi Guellec, Alain Saraux (2017) Methotrexate at the center of the treatment strategy for RA. Rheumatism review monographs. 
7. Lewis HA, Nemer KM, Chibnall RJ, Musiek AC (2017) Methotrexateinduced cutaneous ulceration in 3 nonpsoriatic patients: report of a rare side effect. JAAD Case Rep 3(3): 236-239.

8. Yélamos O, Català A, Vilarrasa E, Roé E, Puig L (2014) Acute severe methotrexate toxicity in patients with psoriasis: a case series and discussion. Dermatology 229(4): 306-309.

9. Truchuelo T, Alcántara J, Moreno C, Vano Galván S, Jaén P (2010) Focal skin toxicity related to methotrexate sparing psoriatic plaques. Dermatol Online J 16(6): 16.

10. Methotrexate (2010) Clinical Pharmacology On-Line. Gold Standard, Inc. Available at www.clinicalpharmacology. com.
11. Fridlington JL, Tripple JW, Reichenberg JS, Hall CS, Diven DG (2011) Acute methotrexate toxicity seen as plaque psoriasis ulceration and necrosis: a diagnostic clue. Dermatol Online J 17(11): 2.

12. Tan KW, Tay YK (2011) A case of acute methotrexate toxicity. Ann Acad Med Singap 40(2): 97-99.

13. Gilani STA, Khan DA, Khan FA, Ahmed M (2012) Adverse effects of low dose methotrexate in rheumatoid arthritis patients. J Coll Physicians Surg Pak 22(2): 101-104 